ARTICLE

\title{
Feasibility Study on the Review Framework for the Radiation Safety of Shielding Designs by Use of MCNP
}

\author{
Byeong-Soo KIM* \\ Korea Institute of Nuclear Safety \\ 19 Kusong-Dong, Yusong-Ku, Daejeon, 305-338, Republic of Korea
}

\begin{abstract}
Pre-conceptual review framework is developed for the radiation shielding safety of MCNP-based shielding designs and its feasibility study is performed, mainly focused on the capability of the review framework for the verification of the right use of MCNP. General review items and MCNP code-specific review items are separately implemented in review procedures. The feasibility of the review framework including MCNP code-specific review items is studied and identified via the application of the case study. MCNP code-specific review items are considered as pass of ten statistical checks, identification of violation of physical consistency and activities of independent analysis. It is seen that MCNP code-specific review items are useful to verify the right use of MCNP features such as statistical characteristics and variation reduction techniques.
\end{abstract}

KEYWORDS: review framework, feasibility study, radiation shielding, MCNP, variance reduction, statistical characteristics, physical consistency, independent analysis, Monte Carlo simulation

\section{Introduction}

Radiation shielding designs are often performed by use of $\mathrm{MCNP}^{1)}$ code system, based on the Monte Carlo simulation. It is more preferred nowadays than before, due to its continuously updated capabilities and the natural 3-dimensional simulation to evaluate particle properties.

There are some weak points on the MCNP code system, arising from its statistical behaviors of results and the needs of variance reductions. Variance reduction techniques ${ }^{2)}$ are often necessary to be utilized to get reliable results whose uncertainties coming from the statistical behaviors are minimized. But, sometimes, wrong uses of the MCNP features such as variance reduction techniques might result in wrong answers. In this study, mainly focused on the capability of the review framework for the verification of the right use of MCNP, feasibility study is performed and pre-conceptual review framework is developed for the radiation shielding safety of MCNP-based shielding designs.

\section{Review Framework for the Radiation Shielding} Safety

\section{Review Framework}

Pre-conceptual review framework is developed for the radiation shielding safety of shielding designs. The overall diagram of review framework is shown in Figure 1. The framework includes acceptance criteria ${ }^{3)}$, review procedures and activities of independent analyses. General review items and code-specific review items are separated in review

*Corresponding Author, E-mail: k484kbs@kins.re.kr

(C) 2012 Atomic Energy Society of Japan, All Rights Reserved. procedures. General and common items such as material composition, geometry modeling, dose conversion factors, appropriateness of input values and ways of homogenization and simplification would be normally and usually reviewed whatever computational codes are utilized. Such items are categorized into the field of general review items. But, items relevant to or dependent on the utilized computational code such as MCNP would be reflected as review items and put in the field of code-specific review items.

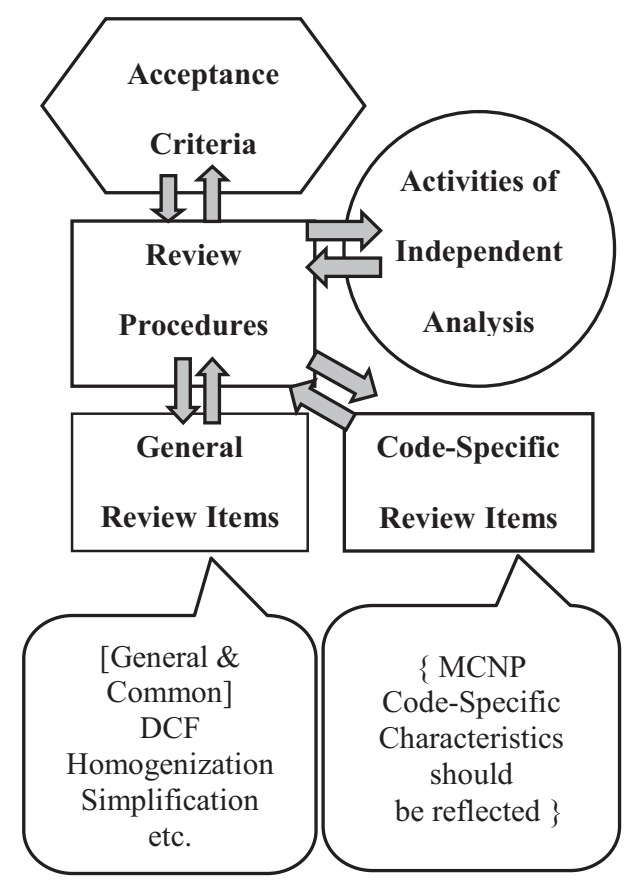

Fig. 1 The overall diagram of review framework 


\section{MCNP code-specific Review Items}

Code-specific review items are filled with MCNP code-specific review items derived by the consideration of MCNP features such as statistical characteristics and variance reduction techniques.

\section{(1) Statistical Characteristics}

The values of particle properties evaluated by MCNP code, based on Monte Carlo simulation, consist of means and relative errors. These are calculated from many independent trials where behaviors of simulated particles are selected and transported according to physical probabilities. They are under statistical characteristics such as central limit theorem and confidence interval.

MCNP code provides 10 statistical checks to assure the reliability of statistical results. Ten statistical checks are made to test the validity of ten statistical behaviors shown by tally fluctuation chart bin of estimated mean, relative error, variance of variance, figure of merit and history tally probability density function. For example, one of 10 checks is a bin error check. Validity of the bin error is checked by comparing relative errors with the recommended ranges such as Relative error $<0.05$ for a point detector tally (F5) and Relative error $<0.1$ for other tallies.

10 statistical checks provided by MCNP are introduced as code-specific review items of the review framework in this study. For MCNP code-specific review items, PASS of 10 statistical checks is placed in the field of code-specific review items.

\section{(2) Variance Reduction Techniques}

To improve precision of MCNP results, variance reduction techniques (VRT) are often applied. Precision is the uncertainty in estimated mean value caused by the statistical fluctuations in the sampled histories and is affected by tally type, number of histories run, forward versus adjoint calculation and VRTs. Precision is different from accuracy whose factors are related to code accuracy, problem modeling and user errors or abuse.

However, sometimes, the wrong use of VRT might corrupt the accuracy of results due to user error or abuse whereas the precision is improved. It is because wrong or abused use of VRT could result in the violation of physical consistency, and the corruption of the accuracy might be followed.

MCNP code-specific review items should be introduced to verify the right use of VRT in MCNP. But, it is not easy to implement systematic and quantitative review items capable of identifying rightness or wrongness of the user activities. Instead, in this study, qualitative review items are introduced as MCNP code-specific review items to verify the right use of the code, such as identification of violation of physical consistency and activities of independent analysis by use of different codes. Although activities of independent analysis are already a main component of review framework, it is additionally placed in the field of code-specific review items to emphasize its importance in the usage of MCNP code.

(3) MCNP code-specific Review Items

From the consideration of statistical characteristics and variance reduction techniques, the followings are derived as MCNP code-specific review items and placed in the field of code-specific review items of the review framework.

- Pass of 10 Statistical Checks

- Identification of Violation of Physical Consistency

- Activities of Independent Analysis

\section{Case Study}

A case study is performed to apply the pre-conceptual review framework. It is mainly focused on the evaluation of feasibilities of MCNP code-specific review items. For this purpose, a simple but deep penetration shielding problem is modeled and simulated by MCNP. Code-specific review items are applied to the case study and the feasibility of the review framework including MCNP code-specific review items is identified.

\section{Simple Deep Penetration Shielding Problem}

A spherical geometry with radius of $40 \mathrm{~cm}$ is modeled for the case study. The sphere is made of a shielding material of a pure lead. A spherical volume source of radius of $2 \mathrm{~cm}$ is located at the center with the gamma energy of $0.9 \mathrm{MeV}$ as shown in Figure 2. The source energy of $0.9 \mathrm{MeV}$ instead of 1 $\mathrm{MeV}$ is selected for the purpose of the simple comparison of results of MCNP with that of ANISN ${ }^{4)}$. The energy near 1 $\mathrm{MeV}$ in the group cross section library, BUGLE- $96^{5)}$ used for ANISN in this study is the $11^{\text {th }}$ group energy of $0.9 \mathrm{MeV}$ within the energy group structure.

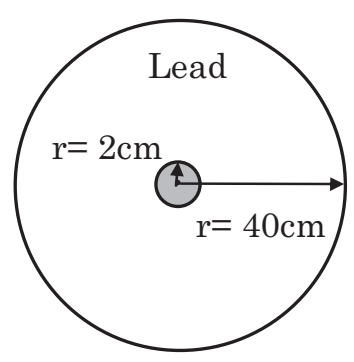

Fig. 2 Geometry of the case study.
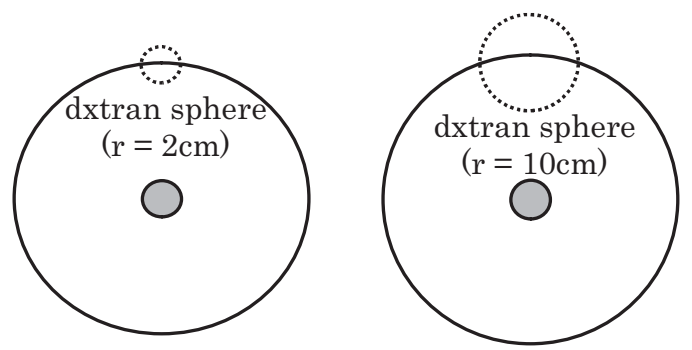

Fig. 3 DXTRAN spheres for v5, v6 $(\mathrm{r}=2 \mathrm{~cm})$ and $\mathrm{v} 7(\mathrm{r}=10 \mathrm{~cm})$.

For 7 different conditions with different tallies and/or VRTs, fluxes at $40 \mathrm{~cm}$, that is, at the surface of the sphere, are evaluated and the code-specific review items are applied for the validity review of MCNP results. The seven cases are as v1 v7 as listed in Table 1. 
Tally of F2 in MCNP can score surface fluence [particles $/ \mathrm{cm}^{2}$ ] whenever a particle crosses the surface such as of the sphere and used in the cases of v1, v3 and v7 as in Table 1. Tally of F4 can score cell fluence [particles $/ \mathrm{cm}^{2}$ ] whenever a particle enters a cell such as the sphere and moves in the cell. The tally is used in the case of v5. Tally of F5 can estimate point detector fluence [particles $/ \mathrm{cm}^{2}$ ] and used in the cases of v2, v4 and v6. F5 tally is usually called as a next-event estimator because it selects randomly a distance moving without collision at the next flight on the basis of physical probability.

VRT (Variation Reduction Technique) is applied to reduce the variance to improve precision of simulated results within reasonable simulation time. In the cases of v1 and v2 listed in Table 1, any VRT is not applied. But, in the cases of v3 and v4, VRT using WWG is applied. WWG (Weight Window Generator) is a useful VRT to effectively reduce variation. It is based on importance of regions. The way of generating of weight windows is that high importance region has small weight windows. When a particle move into a region with a weight window, it might survive and keep moving, be killed or be split according to comparison of the particle weight with that of the region. In the cases of v5, v6 and v7, VRT using DXTRAN is applied. DXTRAN is usually used when angular biasing is needed. DXTRAN sphere is put in the place of interest where angular biasing is to be happened. Whenever collisions happened, DXTRAN sphere attracts a particle from an arbitrary collision point to the surface of DXTRAN sphere and modifies the particle weight.

DXTRANs of radius $2 \mathrm{~cm}$ (for v5 and v6) and $10 \mathrm{~cm}$ (for v7) are applied. The DXTRANs modeled in the cases of v5, v6 and v7 are shown in Figure 3. Although the use of DXTRAN is not recommended in deep shielding problems such as this case study, it is used to show that the wrong use of VRTs can give the wrong answer and the wrong use can be identified within the process of applying the review framework including MCNP code-specific review items.

\section{Pass of 10 Statistical Checks}

Seven different cases of $\mathrm{v} 1 \sim \mathrm{v} 7$ are simulated by MCNP and the fluxes at $40 \mathrm{~cm}$ are evaluated. The results of cases of v1 $\sim$ v7 are shown in Table 1. As in the review procedures, MCNP code-specific review items of 10 statistical checks are applied to the results.

The case of v1 shows all zeros and 10 statistical checks cannot be applied due to no nonzero. It means failure of the MCNP simulation without VRTs and the necessity of use of VRTs. For three cases of v2, v4 and v6, ten statistical checks have performed. But, they have not passed the checks. It can be said that results of $\mathrm{v} 2, \mathrm{v} 4$, and $\mathrm{v} 6$ seem not to be reliable.

For the cases of v3, v5 and v7, they have all passed ten statistical checks. So, the values of them seem to be reliable. However, the values are all different from each other. It is difficult to say which value is the right answer. It means that it is not sufficient to verify the right answer only by testing ten statistical checks provided by MCNP code if VRTs are utilized improperly, though the problem modeling is right. More investigation is necessary to identify the right answer.
Table 1 Result and 10 Statistical Checks.

\begin{tabular}{c|c|c|c|c}
\hline No & $\begin{array}{c}\text { Tally } \\
\text { \& VRT }\end{array}$ & $\begin{array}{c}\text { Flux } \\
\text { at 40cm }\end{array}$ & $\begin{array}{c}\text { Relative } \\
\text { error }\end{array}$ & $\begin{array}{c}\mathbf{1 0} \text { statistical } \\
\text { checks }\end{array}$ \\
\hline v1 & $\begin{array}{c}\text { (F2 tally, } \\
\text { No VRT) } \\
\text { (F5 tally, }\end{array}$ & 0 & 0 & no nonzero \\
v2 & $\begin{array}{c}\text { No VRT) } \\
\text { (F2 tally, }\end{array}$ & $\mathbf{8 . 8 0 8 6 E - 1 9}$ & $\mathbf{0 . 0 1 7 3}$ & PASSED \\
v3 & $\begin{array}{c}\text { WWG) } \\
\text { (F5 tally, }\end{array}$ & $7.4709 \mathrm{E}-19$ & 0.1105 & missed 3 \\
v4 & $\begin{array}{c}\text { WWG) } \\
\text { (F4 tally, }\end{array}$ & $\mathbf{2 . 6 7 7 4 E - 1 9}$ & $\mathbf{0 . 0 4 8}$ & PASSED \\
v6 & $\begin{array}{c}\text { DXT_2) } \\
\text { (F5 tally, } \\
\text { DXT_2) }\end{array}$ & $3.0671 \mathrm{E}-19$ & 0.0263 & missed 3 \\
v7 & $\begin{array}{c}\text { (F2 tally, } \\
\text { DXT_10) }\end{array}$ & $\mathbf{5 . 9 1 5 6 E - 2 1 ~}$ & $\mathbf{0 . 0 3 9 3}$ & PASSED \\
\hline
\end{tabular}

\section{Physical Consistency and Independent Analysis}

Two kinds of MCNP code-specific review items such as identification of violation of physical consistency and activities of independent analysis are applied to the case study. Those are somewhat qualitative items so that the reviewer's experiences and knowledge are required and sufficiently equipped with to verify the right use of VRTs.

Identification of violation of physical consistency can be made by finding caution items that might be listed in the code manuals or addressed by experts and comparing them with the results.

Activities of independent analysis can be performed by making use of other codes whose methodology is different from MCNP, such as the deterministic code. If geometry or specifications of the problem are so complicated that other different code cannot model the problem, ranges such as minimum or maximum of particle properties would be investigated, based on simplification or homogenization, to assure their compliance with the acceptance criteria of the review framework.

Violation of physical consistency is investigated for the case study. Mentioned before, the use of DXTRAN is not recommended in deep shielding problems. But, for three cases of v5, v6 and v7, DXTRAN are made use of in such a deep shielding problem as the case study. It can be a typical improper usage of DXTRAN. So, the results of cases of v5, v6 and v7 might be unreliable.

Independent analysis is performed by ANISN code, based on the one-dimensional deterministic method different from that of MCNP code. Fluxes evaluated by ANISN are compared with three cases of v3, v5, and v7 which have passed ten statistical checks, in Figure 4. The case of v3 shows good agreement with the case of A1 that is results of ANISN. The others are lower than $\mathrm{v} 3$ and A1. The fluxes of the case of v3 seem to be reliable and the right answers.

The reason that results of $\mathrm{v} 5$ and $\mathrm{v} 7$ is underestimated compared with the right answer such as the case of v3 is as follows. Due to the use of DXTRAN, the cases of v5 and v7 transport forcibly direct or uncollided particles from source or collision points into the detector through thick shields. They are missing detections of collided particles that can be the most significant part of the contribution within thick shields. 
It is like without build-up effect. Violations of physical consistency happen in v5 and v7, because build-up effects due to collided particles are neglected because of the wrong use of VRTs such as DXTRAN in deep penetration shielding.

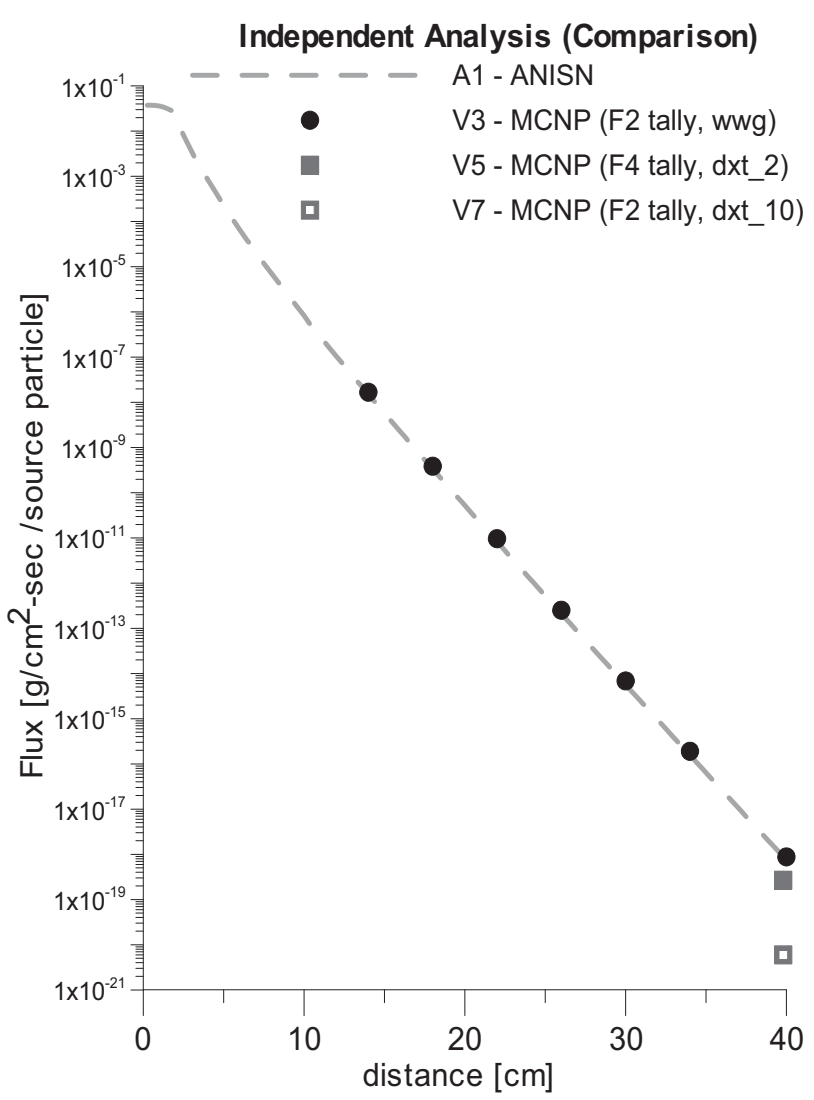

Fig. 4 Independent Analysis and Comparison.

\section{Conclusion}

MCNP code-specific review items are considered as pass of ten statistical checks, identification of violation of physical consistency and activities of independent analysis. Those are introduced as the code-specific review items of review procedures in the review framework for the radiation shielding safety of MCNP-based shielding designs. The feasibility of the review framework including MCNP code-specific review items is studied and identified via the application of the case study.

Through the case study, MCNP code-specific review items are utilized and its feasibility is verified to be achievable. And it is seen that the MCNP code-specific review items are useful to verify the right use of MCNP features such as statistical characteristics and VRTs.

However, some review items except pass of ten statistical checks are not quantitative but qualitative. Further study is necessary to improve qualitative forms of items into more quantitative forms and complete the review framework. It is expected that the set-up of this review framework would satisfy the regulatory demand that might arise in the safety review areas for radiation shielding designs using MCNP code.

\section{Acknowledgment}

This study was supported by the Nuclear Technology R\&D program of the Ministry of Education, Science and Technology (MEST) in Korea.

\section{References}

1) X-5 Monte Carlo Team, "MCNP - A General N-Particle Transport Code, Version 5, Volume I: Overview and Theory", LA-UR-03-1987 (April, 2003).

2) Thomas E. Booth, "A sample problem for Variance Reduction in MCNP", LA-10363-MS (1985).

3) US NRC, Standard Review Plan for Dry Cask Storage Systems, NUREG-1536, 1997.

4) W. W. Engle, Jr., "ANISN, A One-Dimension-al Discrete Ordinates Transport Code with Anisotropic Scattering," K-1693 (March 1967).

5) BUGLE-96 : Coupled 47 Neutron, 20 Gamma-Ray Group Cross Section Library Derived from ENDF/B-VI for LWR Shielding and Pressure Vessel Dosimetry Applications, RSICC Data Library Collection, DLC-185/BUGLE-96 (March 1996) 